\title{
La presencia vivificante de la belleza en la construcción de la interioridad cristiana. Lectura estética del Libro X de las Confesiones de Agustín (1)
}

Puente entre la antigüedad y el cristianismo (2), la estética de Agustín asumió los principios clásicos de ritmo y contraste, para transfigurarlos a la luz de una belleza interior concebida como huella inmemorial de la presencia divina. En esto reconocen los historiadores la piedra fundacional de la estética cristiana (3). Sobre la base de la lectura textual del Libro $X$ de Confesiones realizada en la sección de Filosofía medieval de esta Academia (4), esta ponencia tiene por objeto poner de relieve el papel de la belleza en la hoy urgente reconstrucción de la interioridad como fuente de vida y demostrar así la actualidad de esta vertiente del pensamiento medieval.

La exposición se divide en dos partes. En la primera, se planteará la génesis de los hitos fundamentales de la estética agustiniana hasta Confesiones; los mismos serán considerados en tanto elementos configuradores de la tríada modusspecies-ordo. En la segunda, se analizará la estructura estético formal del Libro $X$ a la luz de los principios descriptos. Esta interpretación se llevará a cabo según el método estético de la totalidad en el fragmento. Por este motivo solo se analizarán los capítulos introductorios (I a VII) en la medida en que representan

(1) Este texto fue presentado por al autora en las Segundas Jornadas Nacionales de Filosofía Medieval, realizadas en la Academia Nacional de Ciencias de Buenos Aires, del 18 al 20 de abril de 2007.

(2) Cfr. SVOBODA, KAREL, La estética de San Agustín y sus fuentes, Madrid, Imprenta Sagrado Corazón, 1958, 327-333.

(3) Los historiadores de la estética coinciden en conferirle este rol fundacional a Agustín. Citamos como ejemplo el siguiente juicio: "Con su obra empieza la estética cristiana de Occidente. [...] San Agustín asumió los principios estéticos de los antiguos, los transformó y los transmitió a la Edad Media. Su obra constituye un punto crucial en la historia de la estética, en el que convergen las corrientes antiguas y de donde derivan las medievales". TATARKIEWICZ, WLADYSLAW, Historia de la estética. II. La estética medieval. Madrid, Akal, 1991, 51. Cfr. DE BRUYNE, EDGAR, Historia de la estética. II. La antigüedad cristiana. La Edad Media, Madrid, Biblioteca de Autores Cristianos, 1963, 267; BODEI, REMO, La forma de lo bello, Madrid, Visor, La balsa de la Medusa, 1998, 35.

(4) En su calidad de director de la Sección de Filosofía Medieval del Centro de Estudios Filosóficos, el Dr. Ricardo Díez organizó un Seminario de lectura del libro X de las Confesiones de Agustín. (Academia Nacional de Ciencias de Buenos Aires, junio-diciembre 2006). 
una anticipación temática y formal del capítulo XXVII, el cual constituye el clímax formal del libro.

\section{HiTOS DE LA GÉNESIS DE LA ESTÉTICA AGUSTINIANA HASTA CONFESIONES}

Considerados por la crítica como reelaboraciones agustinianas de tesis clásicas (5), ritmo y contraste son conceptos que enlazan la estética antigua con la naciente estética medieval. Etimológicamente ritmo es lo que fluye (en griego $\rho v \theta \mu o ́ s$ procede de $\dot{\rho} \varepsilon \omega$, fluir), es decir, lo que sucede en el tiempo siguiendo el "regular retorno de los mismos elementos y estructuras" (6). Dicho estéticamente, posee ritmo lo que está configurado sobre la base de una medida numérica que se reitera proporcionadamente. Para los historiadores, lo propio de la armonía y simetría no radica tanto en el número como en el carácter relacional de la disposición de los elementos que componen la estructura estética (7).

Como los antiguos, Agustín también identifica el concepto de numerus con los de proporción y ritmo, pero introduce variaciones significativas. Primero, extiende a todo objeto estético el concepto de ritmo oriundo del dominio musical (8). Segundo, subraya el principio de razón que rige la relación entre ritmo y belleza, afirmándolo tanto para el orden de la creación como para el de la percepción de la forma bella; es la razón la que comprende que hay belleza donde hay ritmo, es decir, donde hay una relación numérica regida por la igualdad de la repetición (9). Tercero, afirma la necesidad de una simpatía entre el ritmo objetivo de la forma bella y el ritmo subjetivo del alma, lo que lo lleva a desarrollar una "psicología de lo bello" que ha sido evaluada como una de las tesis propias de su estética (10). Si bien es cierto que

(5) Cfr. TATARKIEWICZ, WLADYSLAW, Historia de la estética. II. La estética medieval. Madrid, Akal, 1991, 54.

(6) BODEI, REMO, La forma de lo bello, Madrid, Visor, La balsa de la Medusa, 1998, 32.

(7) BODEI, REMO, La forma de lo bello, Madrid, Visor, La balsa de la Medusa, 1998, 29.

(8) Cfr. TATARKIEWICZ, WLADYSLAW, Historia de la estética. II. La estética medieval. Madrid, Akal, 1991, 54.

(9) En estos términos afirma de Bruyne la razón estética agustiniana: "Comprobamos el orden en el conocer y en el hacer. La facultad mediante la cual comprendemos y creamos el orden es la razón; en el hacer, la razón origina el orden, en el conocer se acomoda al orden. [...] La razón es la actividad de la mente por la cual determinadas adquisiciones aprendidas se separan o se unen entre sí. Racional es, por consiguiente, todo lo que lleva el sello de la razón, pues muestra un orden en el que (merced a la razón) las partes de un todo están diferenciadas claramente y mutuamente adaptadas para originar una sola y única forma. [...] Una percepción por separado no posee nada racional aun cuando halaga al momentáneo deleite. [...] Lo bello, en sentido estricto, es la consonancia racional, medida, proporcional, de diversas partes de un conjunto entre sí. No es posible expresar más exactamente el formalismo pitagórico matemático. [...]. La belleza sensible, por lo tanto, es por naturaleza una ordenación de percepciones, una síntesis de racionalidad y de impresiones sensitivas donde la "forma" resulta decisiva. [...] Rationabile no significa "racional", sino, como ya vimos más arriba, "formado armoniosamente gracias a la razón". DE BRUYNE, EDGAR, Historia de la estética. II. La antigüedad cristiana. La Edad Media, Madrid, Biblioteca de Autores Cristianos, 1963, 274-277.

(10) Cfr. TATARKIEWICZ, WLADYSLAW, Historia de la estética. II. La estética medieval. Madrid, Akal, 1991, 55-56; 
Agustín mantuvo la precedencia objetiva del numerus (11), en el pathos rítmico de su estética ambos ritmos -el objetivo de la belleza y el subjetivo del alma- deben entrar en sintonía para que el juicio estético sea verdadero (12).

El mismo principio estético de orden que da lugar a la proporción es el que rige la diferenciación de las partes produciendo el contraste. En la propuesta agustiniana se armonizan la estética de la igualdad (aequalitas numerosa) con la estética de los contrastes ordenados (13). Ritmo y contraste conforman la base de la tríada musical agustiniana según la cual hay armonía (numerus) donde hay unidad, igualdad y ordenación.

A partir de su conversión al cristianismo y bajo la influencia del texto de Sabiduría 11, 21 -“Todo lo hiciste, Señor, ordenado según medida, número y peso"-, Agustín desarrolla una estética sapiencial (mensura-numerus-pondus), que deriva finalmente en la formulación trinitaria modus-species-ordo, base de la idea general de belleza que aparece en Confesiones (14). Si bien los historiadores coinciden en

(11) “[...] gracias al gusto subjetivo, gozamos espontáneamente del ritmo objetivo. Pero entonces se plantea la pregunta siguiente: ¿qué es lo que apreciamos en la armonía sensitiva? Y la respuesta es clara: la "igualdad" de las partes dentro del conjunto. [...] El espíritu se siente embelesado por la igualdad y la proporción; allí donde encontremos las formas bellas, aunque no realicen la igualdad ideal, será porque la imitan. [...] La idea de orden viene a completar las nociones de igualdad y semejanza, que se apoyan a su vez en la idea de unidad. Y asíllegamos a un primer ternarium filosófico: Toda armonía (numerus) es unidad, igualdad y ordenación. [...] Principio de la armonía es la unidad, su consecuencia es la igualdad; forma suprema de la proporción y lazo de unión entre las partes proporcionadas entre sí es el orden... ¿Interpoló Agustín este pasaje con ciertas consideraciones sobre la Santísima Trinidad en su revisión posterior de la obra? [...] Donde encontramos igualdad y semejanza, allí habrá armonía. Y donde sentimos espontáneamente la presencia de la armonía, que acomoda la conciencia al objeto y el objeto a la conciencia, allí gozamos de la belleza... [...] A la armonía de las formas externas corresponde la armonía de la vida del alma. [...] La belleza radica en la convenientia mutua entre sujeto y objeto." DE BRUYNE, EDGAR, Historia de la estética. II. La antigüedad cristiana. La Edad Media, Madrid, Biblioteca de Autores Cristianos, 1963, 290-296.

(12) Cfr. Confessiones, X, VI, 10. "Agustín desarrolla aquí una psicología completa y detallada de la que retenemos que, en última instancia, todas las armonías se realizan merced a la actividad del alma. [...] Por lo que respecta al ritmo de la percepción, ofrécese aquí una coincidencia o concordancia entre el sonido externo y la actividad interna. El fin que se propone Agustín es demostrar la primacía de lo psicológico sobre lo físico. Sin incitación alguna externa, nos dice, la psique anima y vivifica al oído, produciendo en él movimientos vitales llenos de ritmo. Cuando un sonido afecta al tímpano o membrana del oído, este observa inmediatamente los cambios operados en el órgano. Si el sonido se muestra acorde con su propia armonía vital, gozará de la facilidad con que ambas cosas se unifican en la percepción y experimentará el placer de la audición. Los ritmos de la memoria fluyen también de la armonía vital del alma. [...] De todo esto puede deducirse que para Agustín el sentimiento musical o, mejor dicho, todo el sentimiento estético, es una síntesis armoniosa de varios ritmos físicos y psicológicos. Una meditación más detenida sobre este particular nos hace ver que el juicio estimativo sensible difiere de la crítica científicamente fundamentada. El goce permanece invariable, pero en un caso es aceptado instintiva e impremeditadamente y en el otro la razón hace saber por qué el uno agrada justamente y el otro no. Los ritmos de la actividad crítica o enjuiciadora deben ser reservados para la razón espiritual." DE BRUYNE, EDGAR, Historia de la estética. II. La antigüedad cristiana. La Edad Media, Madrid, Biblioteca de Autores Cristianos, 1963, 288-289.

(13) Cfr. DE BRUYNE, EDGAR, Historia de la estética. II. La antigüedad cristiana. La Edad Media, Madrid, Biblioteca de Autores Cristianos, 1963, 297-304.

(14) Cfr. SVOBODA, KAREL, La estética de San Agustín y sus fuentes, Madrid, Imprenta Sagrado Corazón, 1958, 213; DE BRUYNE, EDGAR, Historia de la estética. II. La antigüedad cristiana. La Edad Media, Madrid, Biblioteca de Autores Cristianos, 1963, 312. 
señalar la imprecisión de los límites de la terminología agustiniana (15), se puede establecer una correspondencia entre los pares bíblico-estéticos mensura-modus, numerus-species y pondus-ordo.

El primer par, mensura-modus, dice relación al hecho genérico de que el modo de ser de lo bello está constituido por la unidad y límite de la forma. El segundo par, numerus-species, no se refiere a la determinación metafísica sino a la manifestación concreta de la forma en una figura sensible regida por el ritmo y el contraste. El tercer par, pondus-ordo, apunta al amor como principio de unidad de un todo donde cada parte encuentra su lugar (16). Para Agustín, la fuente de estas notas estéticas es Dios: Él es quien todo lo delimita otorgando a cada uno su propia medida o modo de ser (mensura-modus), quien todo lo configura en armonía de ritmos iguales y de contrastes (numerus-species), quien todo lo dispone según el peso de su amor (pondus-ordo).

En el libro décimo de Confesiones esta fórmula, la más representativa de la estética agustiniana (17), se enlaza con el dinamismo memorial del hombre espiritual que descubre en la belleza interior la huella inmemorial de la belleza divina originaria (18). Esto se traduce en la propuesta de una estética viva e itinerante, regida no por un estatismo numérico y esencialista propio del dualismo platónico entre sensibilidad y espíritu, sino por un "orden en marcha”, que se encuentra "en tensión dinámica hacia una unidad insequible": la de la belleza divina (19).

(15) Cfr. DE BRUYNE, EDGAR, Historia de la estética. II. La antigüedad cristiana. La Edad Media, Madrid, Biblioteca de Autores Cristianos, 1963, 315.

(16) Cfr. DE BRUYNE, EDGAR, Historia de la estética. II. La antigüedad cristiana. La Edad Media, Madrid, Biblioteca de Autores Cristianos, 1963, 313-315.

(17) Para evaluar el alcance histórico de esta formulación bástenos citar este juicio del ya mencionado investigador polaco: "San Agustín apoyó esta teoría: "Solo la belleza agrada; y dentro de la belleza, las figuras; en las figuras, las proporciones; y en las proporciones, los números" ( $D e$ ordine, II, 15, 42). Ningún griego clásico había explicado nunca esta vieja idea helénica con más énfasis que el padre de la Iglesia. "No hay ninguna cosa ordenada que no sea bella" (De vera religione, XLI, 77). Y de nuevo: "Las cosas bellas agradan en virtud del número que contienen en sí mismas" (De musica, VI 12,38). Y finalmente: "Cuanta más medida, figura y orden haya en las cosas, mayor será su valor" (De natura boni, 3). Esta tríada (modus, species, ordo) se convirtió en una fórmula de la estética medieval y con ella sobrevivió durante mil años." TATARKIEWICZ, WLADYSLAW, Historia de seis ideas. Arte, belleza, forma, creatividad, mímesis, experiencia estética, Madrid, Tecnos, 1992, 256-257.

(18) Cfr. DE BRUYNE, EDGAR, Historia de la estética. II. La antigüedad cristiana. La Edad Media, Madrid, Biblioteca de Autores Cristianos, 1963, 326-327.

(19) Tanto Balthasar desde su perspectiva estético-teológica como Bodei, desde su punto de vista estético filosófico, coinciden en afirmar el carácter dinámico de la estética de Agustín. Dice Balthasar que en Agustín, la armonía del mundo no se construye "sobre relaciones numéricas fijas en el tiempo y espacio como algo definitivamente dado, en cuya visión pasiva el espíritu contemplativo descubre sentimientos estéticos. Para Agustín, esta belleza es más bien una tensión dinámica hacia una unidad inasequible, que es en todo caso espiritual, y en definitiva divina y hace trascender ónticamente la belleza numérica." BALTHASAR, HANS URS VON, Gloria. Una estética teológica. 2. Estilos eclesiásticos, Madrid, Encuentro, 1986, 130. Por su parte, señala Bodei al respecto: "Cuando se refiere al mundo como "poema del universo" (La música VI, 11, 29), es decir, cuando lo compara con una obra de arte, Agustín introduce la voluntad y el carácter imprevisible de un artista divino, creador desde la nada, en el orden rígido y eterno de los griegos. Como el orden de la civitas Dei peregrinans - de la comunidad de fieles en viaje desde la existencia terrena hacia el paraíso- el suyo es un orden en marcha, un puente lanzado sobre el abismo con el objeto de unir lo humano y lo divino el tiempo y la eternidad." BODEI, REMO, $L a$ forma de lo bello, Madrid, Visor, La balsa de la Medusa, 1998, 36. 
2. Belleza, interioridad y vida en la estructura estética del Libro $X$ de Confesiones

Lo que nos proponemos demostrar en esta segunda parte es que la estructura del Libro X es estética no solo por su contenido -por la belleza del alma a la Belleza divina, por la inmanencia a la trascendencia-, sino también por su forma, la cual responde a los principios de ritmo, contraste y belleza interior que conforman -como ya señalamos- la base de la fórmula modus, species, ordo.

En el conjunto de la obra, el Libro $X$ representa un giro significativo en el curso del relato confesional, ya que la voz del narrador cambia la perspectiva pretérita de los libros anteriores para situarse -y situarnos a nosotros sus lectores- en el escenario de un presente dialógico y dramático, donde la acción divina y la humana se entreveran para configurar el mundo vital de la interioridad personal. El tema que atraviesa este libro es la pregunta existencial por el “¿quién soy yo?”. El propósito de la confesión de Agustín es encontrar la respuesta existencial a esta pregunta. Hasta aquí lo hizo mirando hacia el pasado, a partir de aquí lo hará anclado en el presente de su escritura. Así lo dice expresamente en dos pasajes introductorios: "confiteor [...] quis ego sim, non quis fuerim" (III, 4); "hic est fructus confessionum mearum, non qualis fuerim, sed qualis sim" (IV, 6).

Al cambio de perspectiva narrativa y de orientación temática -de la memoria en el tiempo pasa a la memoria de la fuente inmemorial que habita en el centro del hombre- le corresponde un consecuente cambio de ritmo y de forma. Es justamente aquí donde la via pulchritudinis aparece como la forma que más adecuadamente expresa el origen divino inmemorial que habita en lo profundo de la interioridad humana.

La figura (species) del primer capítulo está ordenada según al ritmo estético de la simetría y el contraste. Tres son los modos en los que el ser de Dios se hace presente en el yo de Agustín: conocimiento, fuerza y amor: "cognoscam te, cognitor meus", "virtus anima mea, intra in eam", "veritatem dilexisti [...] venit ad lucem" $(I, 1)$. El viaje por la memoria en busca de la belleza inmemorial que está por emprender responde a este ritmo triádico. Esta forma ternaria del ritmo de la acción en el tiempo se entrecruza inmediatamente con la forma binaria del contraste entre lo vertical y lo horizontal, cuando el narrador señala que hará su confesión ante Dios "coram Te" y ante los hombres "coram multis testibus" (I, 1). Por medio del contraste se configura el escenario espacial, por medio del ritmo discursivo se pauta el movimiento de las acciones en el tiempo.

Los capítulos subsiguientes no harán sino desplegar el orden de estas figuras o species que son expresión de modos o formas de ser. Mientras el capítulo II desarrolla el tema vertical (coram Te), el capítulo III despliega el horizontal (coram multibus testibus). Este contraste queda reforzado estructuralmente por el dinamismo de descenso y de ascenso representado temáticamente por la manifestación del abismo de la propia conciencia de los capítulos I, II y III por un lado y el IV por otro.

En figura de ritmo y contraste se va configurando el dinamismo del viaje hacia la más íntima intimidad. La belleza irrumpe en el marco de una confesión hecha en un espacio interior al que, desde la perspectiva de nuestro horizonte de sentido actual, proponemos interpretar como metáfora teatral. Así, tras el prólogo de los 
primeros cuatro capítulos (en cuyo "espacio vacío" (20) se produce, por un lado, la entrada de los actores: el narrador que confiesa y Dios que actúa; y, por otro, la aparición del público: los muchos testigos o destinatarios), y luego del "estásimo" o momento contemplativo del capítulo quinto, acontece la acción propiamente dicha: en los capítulos sexto y séptimo se explicitan los elementos de la figura estética agustiniana según la tríada modus-species-ordo. Aquí es donde aparece el tema de la búsqueda y del encuentro de Dios en la hermosura.

Si realizamos una lectura textual del capítulo VI, advertimos que el escenario donde acontece la acción rítmica de búsqueda y encuentro, de pregunta y respuesta, es el interior del corazón (21). "Amo te amavi te" (VI, 8): el capítulo se inicia planteando el tema central del encuentro de amor en la forma del contraste del tiempo pasado con el tiempo presente. En este marco se subraya el hecho de que la acción humana es respuesta al amor de Dios que amó primero, hiriendo y penetrando "percussisti cor meum verbo tuo" (VI, 8). La conclusión de Agustín es decisiva: el lugar de la revelación de Dios es la belleza que el hombre descubre en su interior.

En los capítulos VI y VII, una y otra vez retorna rítmicamente la pregunta: "Quid autem amo, cum te amo?" (VI, 8); "Et quid est hoc?" (VI, 9); "Quid ergo amo, cum Deum meum amo?" (VII, 11). La respuesta al objeto del amor coincide con la respuesta al "quién soy yo". El camino que recorre Agustín hasta encontrar una respuesta a esta pregunta existencial es -reiteramos- la via pulchritudinis.

El ritmo binario de pregunta y respuesta constituye el dinamismo de la búsqueda agustiniana: "Interrogatio mea intentio mea et responsio eorum species eorum” (VI, 9). La respuesta se va configurando a partir del contraste. El primer movimiento es apofático, a Dios no lo encuentra en el afuera sensible: "non speciem corporis", "non decus temporis" (VI, 8). El segundo movimiento es katafático, a Dios lo encuentra en el adentro espiritual: "Et tamen amo quandam lucem et quandam vocem et quandam odorem et quandam cibus et quandam amplexum, cum amo Deum meum, lucem, vocem, odorem, cibum, amplexum interioris hominis mei" (VI, 8). Aunque es de orden espiritual, la revelación divina en la belleza interior conserva cierto "quandam" aspecto de figura, cierta memoria de luz, voz, olor, gusto y abrazo.

En esta species o belleza interior, Agustín encuentra simultáneamente a Dios y a sí mismo. Concebida en armonía con la medida existencial (modus) y el orden (ordo) la figura (species) no es meramente externa sino aparición de la forma ontológica según el orden existencial del amor. Para que esto suceda no solo hay que interrogar a la belleza sino juzgarla, lo cual supone que el ritmo interior entre en sintonía con el ritmo exterior. La fuente de este ritmo interior es, para Agustín, la vida de Dios que es "vida de la vida" (VI, 10). Este origen le otorga al itinerario agustiniano un dinamismo que no es de rígida unidad sino de unidad en marcha

(20) Aplicamos aquí el conocido concepto brookiano que dice: "Puedo tomar cualquier espacio vacío y llamarlo un escenario desnudo. Un hombre camino por este espacio vacío mientras otro le observa, y esto es todo lo que se necesita para realizar un acto teatral." BROOK, PETER [1968], El espacio vacío. Arte y técnica del teatro, Barcelona, Península, 2000, 5.

(21) Sobre la polaridad existencial entre altura e interioridad cfr. GUARDINI, ROMANO, Mundo y persona, Madrid, Guadarrama [1954], 1963, 69-79, 93-95. 
hacia el Otro como fuente vital y hacia los otros como miembros de una comunidad que recibe la confesión en el amor (III, 3-4).

El Dios que es fuente de esta belleza concebida de acuerdo con la tríada modus-species-ordo es, pues, un Dios vivo. Para Balthasar, este Dios vivo que hace perceptible su vitalidad en la belleza es el centro de la estética de Agustín (22). La vida divina está regida por el ordo amoris en cuya base alienta la ausencia de envidia que es nota distintiva de lo bello y de la vida por ser exceso de gratuidad que se derrama. La mediación dinámica del amor se opone al dualismo estático entre sensibilidad y espíritu (23) y orienta el movimiento del Libro X hacia el clímax del encuentro con la hermosura.

En efecto, la estructura de estos siete capítulos introductorios al viaje por la memoria interior (VIII-XXVI) anticipan la forma quiásmica de la exclamación con que se inicia el capítulo XXVII. "Sero te amavi pulchritudo tam antiqua et tam nova, sero te amavi!" (XXVII, 38). Reconocemos aquí el hiato entre el tiempo y el instante atemporal de la intuición, entre la antigüedad del dolor y la novedad de la salvación. El adverbio "tarde" (sero) hace alusión a la nostalgia temporal o dolor por el deseo de regresar a la fuente que es la atemporalidad de la hermosura de Dios, que se expresa aquí en la forma finita de la simetría estructural del "tam antiqua - tam nova".

Primero, entonces, la antigua memoria del alejamiento de Dios expresada en la figura de pares de opuestos que se reiteran con orden simétrico ordenadamente: "intus eras - ego foris"; "formosa - deformis"; "mecum eras - tecum non eram" (XXVII, 38). Luego, la novedad de la acción de Dios que irrumpe y sigue irrumpiendo, expresada aquí en la paradoja, cuyos términos ya no están yuxtapuestos sino unidos por el coordinante:

"Vocasti, clamasti - et rupisti surditatem meam;

[Llamaste y clamaste, y rompiste mi sordera;]

Coruscasti, splenduiste - et fugaste caecitatem meam;

[Brillaste resplandeciente, y fugaste mi ceguera;]

Fraglasti - et duxi spiritum et anhelo tibi;

[Exhalaste tu perfume y respiré, y suspiro por ti;]

Gustavi et - esurio et sitio;

[Gusté de ti, y estoy hambriento y sediento;]

Tetigisti me, - et exarsi in pacem tuam.

[Me tocaste, y me inflamé en tu paz.] (XXVII, 38).

(22) Cfr. BALTHASAR, HANS URS VON, Gloria. Una estética teológica. 2. Estilos eclesiásticos, Madrid, Encuentro, 1986, 104.

(23) Cfr. BALTHASAR, HANS URS VON, Gloria. Una estética teológica. 2. Estilos eclesiásticos, Madrid, Encuentro, 1986, 130. 
Esta es la huella de la presencia inmemorial. Hubo un encuentro y la huella ha quedado grabada en el corazón en figura de vida y belleza. El mismo ritmo ternario de conocimiento, fuerza y amor de la introducción sustenta aquí el dinamismo de la presencia y la memoria. Que la infinitud de la presencia inmemorial se exprese en el ritmo finito de la figura bella o species es, para Balthasar, el orden propio de la estética cuyo dinamismo se define, según el teólogo, por las consonancias entre el pathos de la medida rítmica y el pathos de la infinitud (24).

En el comienzo de la era cristiana, la construcción de la interioridad fue tarea de santos teólogos que vieron en la belleza el camino más directo hacia el Dios vivo. Andar tras sus huellas es hoy para nosotros que vivimos en una era postcristiana de eclipse de Dios, una posibilidad de reconstrucción de la interioridad cristiana, conscientes de que proteger la belleza significa proteger la interioridad de la vida. Con los versos de Juan de la Cruz, volvamos también nuestras miradas hacia la fuente originaria y oremos con los versos del Cántico: ";Oh cristalina fuente, / si en esos tus semblantes plateados / formases de repente / los ojos deseados / que tengo en mis entrañas dibujados!” (XXVII, 38).

\title{
RESUMEN
}

A partir del análisis estético del Libro $X$ de Confesiones de Agustín, el propósito de la autora es poner de relieve el papel de la belleza en la hoy urgente reconstrucción de la interioridad como fuente de vida y demostrar así la actualidad de esta vertiente del pensamiento medieval. La fórmula estética modus-species-ordo es presentada como resultado de la integración de los principios pitagóricos de ritmo y contraste con la novedad cristiana de la belleza interior, considerada como sede de la Hermosura y Vida divinas. Belleza, vida e interioridad son propuestos como cimientos para la renovación del itinerario cristiano del siglo XXI.

Palabras clave: Agustín, belleza, interioridad, vida.

\begin{abstract}
Using as her starting point an esthetic analysis of Book X of Augustine's Confessions, the author's purpose is to bring out in relief the role of beauty in today's urgent reconstruction of interiority as a source of life, and demonstrate in this way the relevance for our times of this wellspring of medieval thought. The esthetic formula modus-species-ordo is presented as the result of the integration of the Pythagorean principles of rhythm and contrast with the Christian novelty of interior beauty, considered as the seat of Divine Beauty and Life. The author proposes that beauty, life and interiority constitue the foundation for the renewal of the Christian itinerary of the $21^{\text {st }}$ century.
\end{abstract}

Key words: Augustine, Beauty, Interiority, Life.

(24) Cfr. BALTHASAR, HANS URS VON, Gloria. Una estética teológica. 2. Estilos eclesiásticos, Madrid, Encuentro, 1986, 138. 